\title{
TEM Analysis of Tunnel-Junction Structures in FeCo Films on GaAs Grown by Molecular Beam Epitaxy
}

\author{
Stuart McKernan, Chang Sun Kong, Xuying Dong, and C.J. Palmstrøm \\ Chemical Engineering and Material Science, University of Minnesota, Minneapolis, MN 55455.
}

In the ever-expanding world of semiconductor nanotechnology, epitactic metal films have an important role to play, particularly for metallic or semi-metallic contacts to the semiconductor [1,2]. The growth process of the films and the structure of the complete multilayer assembly after processing need to be carefully characterized to ensure that the desired structure has been successfully fabricated. In this paper we report on the TEM observations of a magnetic tunnel junction consisting of the following structure: $\mathrm{Fe}_{0.5} \mathrm{Co}_{0.5}$ $(\sim 200 \AA) / \mathrm{Al}_{2} \mathrm{O}_{3}(8 \AA) / \mathrm{Fe}(\sim 50 \AA)$ films on III-V semiconductor (GaAs). The $50 \AA \mathrm{Fe}$ film was single crystal and epitactic with the underlying GaAs substrate on which it is grown. A thin layer of aluminum was deposited on top of this Fe layer and oxidized in-situ to form an alumina tunneling barrier about $8 \AA$ wide. This layer obviously needs to be very uniform and pinhole-free in order to function effectively as a tunneling barrier. After the barrier layer is oxidized the polycrystalline $\mathrm{Fe}_{0.5} \mathrm{Co}_{0.5}$ metal overlayer is deposited. The whole structure is finally capped with another aluminum layer to protect the tunnel-junction structure from further, undesired oxidation.

The tunnel-junction structure was characterized using a FEI Tecani F30 field-emission gun transmission electron microscope operating at $300 \mathrm{kV}$. The samples were prepared by tripod polishing followed by a short ion-milling to optimize the specimen thickness at the interfaces. Conventional bright-field imaging (figure 1) reveals that the basic, as-designed structure has indeed been fabricated. The different layers appear to be smooth and uniform; the GaAs substrate on the extreme left, the $5.5 \mathrm{~nm}$ single crystal Fe layer next and even the very thin tunnel barrier which appears to have no pinholes. The Fe Cr overlayer on the right of the image is polycrystalline as expected, and the capping layer appears on the extreme right of the image. Highresolution imaging of the multilayer structure (figure 2) also shows a very clean set of interfaces between the different layers that appear to be abrupt on the atomic scale. There is a small amount of variability in the width of the Fe epilayer, and a larger variability in the thin alumina tunnel barrier. This is not unexpected since the oxidation of the aluminum layer is not as directly controllable as the epilayer deposition processes. The thickness of the layer is measures at $1.0 \mathrm{~nm}+/-0.2 \mathrm{~nm}$ over $50 \mathrm{~nm}$ length scales. Using the small-probe capability of the FEGTEM elemental linescans across these layers were performed (figure 3). The results show that the composition profiles support the basic designed structure. The vertical marker is in the same position in each image, showing the abrupt transition from the pure iron layer to the $\mathrm{Fe}_{0.5} \mathrm{Co}_{0.5}$ layer, and represents the position of the tunnel barrier layer. The presence of both $\mathrm{Al}$ and $\mathrm{O}$ signals from the tunnel barrier layer indicates that the layer is indeed alumina. Several factors affect the sharpness of these profiles, including the actual chemical abruptness of the interfaces, specimen drift during the measurement, the EDX background signal, and the size of the tails on the incident electron probe. Although the alumina tunneling junction is seen to be fully oxidized and less than $2 \mathrm{~nm}$ in width in the HREM images, the composition profiles do not indicate an equally sharp interface. In particular the Fe profile extends through the tunnel barrier into the FeCo layer with no decrease in intensity at the barrier layer. Analysis of these tunnel barriers in terms of the chemical abruptness in both the EDX composition profiles and the HREM images with implications for the characterization of nanoscale materials will be presented. [3]

\section{References}

1. Hilton, J. L., Schultz, B. D., McKernan, S., Spanton, S. M., Evans, M. M. R., Palmstrøm,C. J., 2005, J. Vac. Sci. Technol. B 23(4), 1752-1758.

2. Dong, J. W.; Chen, L. C., Xie, J. Q., 1Müller, T. A. R., Carr, D. M., Palmstrøm, C. J., 
McKernan, S, Pan, Q., James, R. D., 2000, J. Appl. Phys. 88, 7357 - 7359

3. Support was provided by the University of Minnesota Characterization Facility and through NSF grant number DMR-0320641
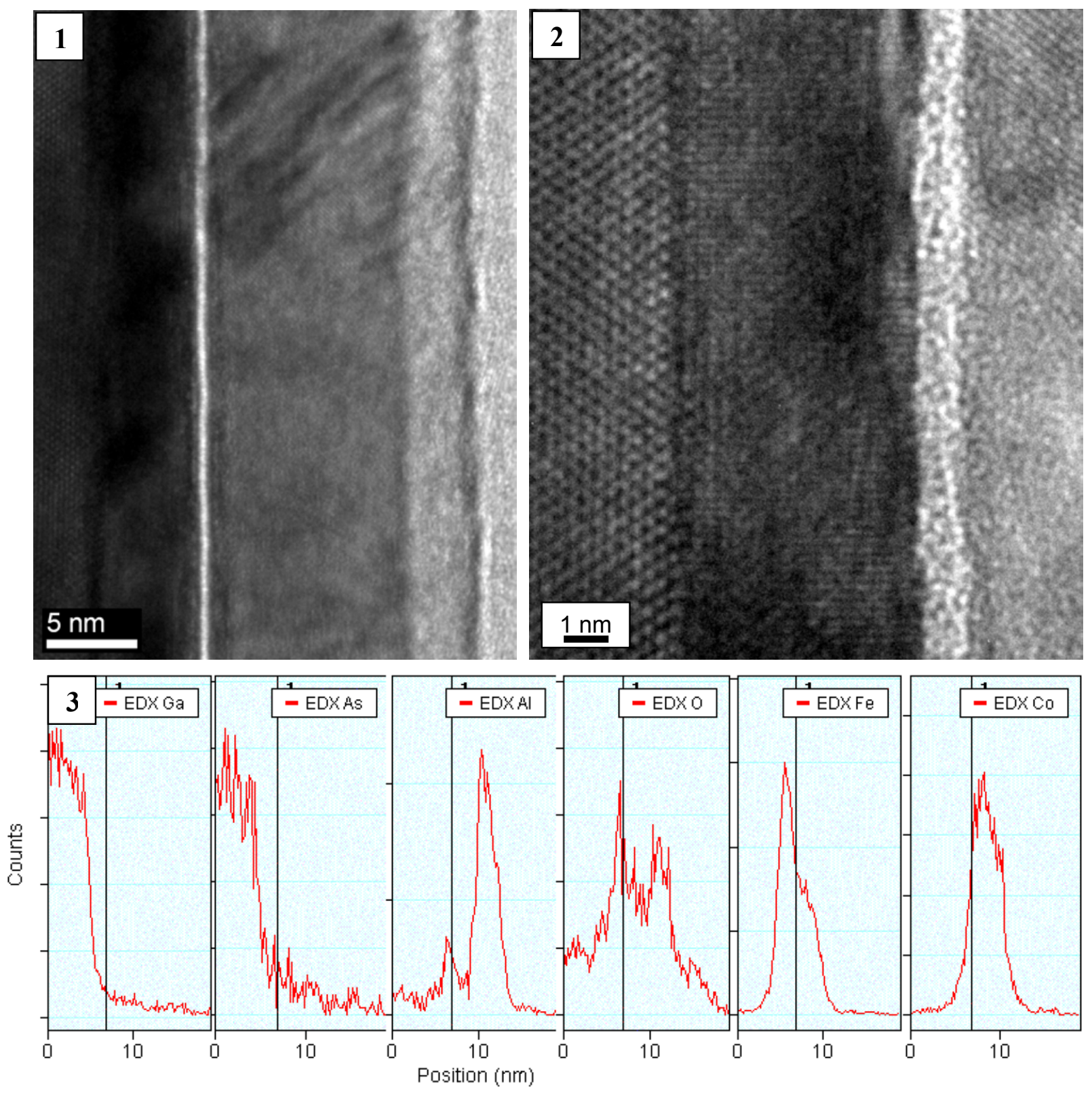

Figure 1 Low magnification bright-field image of the tunnel junction structure, showing the thin alumina buffer layer images as a thin vertical bright feature.

Figure 2 High-resolution image of the device showing uniform layer thicknesses and good epitaxy between the GaAs (leftmost layer) and the single crystal Fe and relative uniformity of the tunnel barrier. Figure 3 STEM EDX profiles across the tunnel junction structure. 